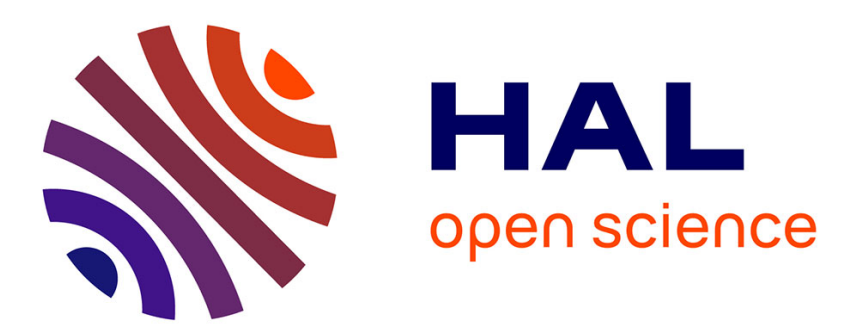

\title{
Necessary and sufficient condition for local exponential synchronization of nonlinear systems
}

Vincent Andrieu, Bayu Jayawardhana, Sophie Tarbouriech

\section{To cite this version:}

Vincent Andrieu, Bayu Jayawardhana, Sophie Tarbouriech. Necessary and sufficient condition for local exponential synchronization of nonlinear systems. 54th IEEE Conference on Decision and Control (CDC 2015), Dec 2015, Osaka, Japan. pp.2981 - 2986, 10.1109/CDC.2015.7402586 . hal-01856301

\section{HAL Id: hal-01856301 https://hal.laas.fr/hal-01856301}

Submitted on 10 Aug 2018

HAL is a multi-disciplinary open access archive for the deposit and dissemination of scientific research documents, whether they are published or not. The documents may come from teaching and research institutions in France or abroad, or from public or private research centers.
L'archive ouverte pluridisciplinaire HAL, est destinée au dépôt et à la diffusion de documents scientifiques de niveau recherche, publiés ou non, émanant des établissements d'enseignement et de recherche français ou étrangers, des laboratoires publics ou privés. 


\title{
Necessary and sufficient condition for local exponential synchronization of nonlinear systems
}

\author{
Vincent Andrieu, Bayu Jayawardhana, Sophie Tarbouriech
}

\begin{abstract}
Based on recent works on transverse exponential stability, some necessary and sufficient conditions for the existence of a (locally) exponential synchronizer are established. We show that the existence of a structured synchronizer is equivalent to the existence of a stabilizer for the individual linearized systems (on the synchronization manifold) by a linear state feedback. This, in turns, is also equivalent to the existence of a symmetric covariant tensor field which satisfies a kind of Lyapunov inequality. Based on this property, we provide the construction of such synchronizer.
\end{abstract}

\section{INTRODUCTION}

Controlled synchronization, as a coordinated control problem of a group of autonomous systems, has been regarded as one of important group behaviors. It has found its relevance in many engineering applications, such as, the distributed control of (mobile) robotic systems, the control and reconfiguration of devices in the context of internet-of-things, and the synchronization of autonomous vehicles.

For linear systems, the solvability of this problem and, as well as, the design of controller thereof, have been thoroughly studied in literature. To name a few, we refer to the classical work on the nonlinear Goodwin oscillators [9], to the synchronization of linear systems in [17], [15] and to the recent works in nonlinear systems [13], [8], [7], [6], [14]. For linear systems, the solvability of synchronization problem reduces to the solvability of stabilization of individual systems by either an output or state feedback. It has recently been established in [17] that for linear systems, the solvability of the output synchronization problem is equivalent to the existence of an internal model, which is a well-known concept in the output regulation theory.

The generalization to nonlinear systems has appeared recently in literature (see, for example, [13], [8], [7], [6], [14], [11]). In these works, based on the concept of passivity theory (or, the weakened notions of co-coercive systems), some sufficient conditions are studied that can be used to construct a synchronizer that solves the synchronization problem. For such a class of systems, the synchronizer is

This work was supported by the ANR Project LIMICOS contract number 12BS0300501

V. Andrieu is with Université de Lyon, F-69622, Lyon, France; Université Lyon 1, Villeurbanne; CNRS, UMR 5007, LAGEP, 43 bd du 11 novembre, 69100 Villeurbanne, France and with Fachbereich C - Mathematik und Naturwissenschaften, Bergische Universität Wuppertal, Gaußstraße 20, 42097 Wuppertal, Germany. vincent.andrieu@gmail.com

B. Jayawardhana is with ENTEG, Faculty of Mathematics and Natural Sciences, University of Groningen, the Netherlands, bayujw@ieee.org, b.jayawardhana@rug.nl

$\mathrm{S}$. Tarbouriech is with CNRS, LAAS, 7 avenue du Colonel Roche, F31400 Toulouse, France and Univ de Toulouse, LAAS, F-31400 Toulouse, France.tarbour@laas.fr. constructed based on the relative output/state measurement, as in the linear systems case. In [10], small-gain theorem is used to construct a synchronization control law for $L_{2}$-stable systems. If we restrict ourselves to the class of incremental ISS, as discussed in [4], the synchronizer can again be based on the relative output/state measurement.

In general, with the lack of characterization of controlled synchronization for general nonlinear systems, it is difficult to conclude on the generality of the synchronizer as proposed in the aforementioned works. Using recent results on the transverse exponential contraction, we establish in this paper some necessary and sufficient conditions for the solvability of a (locally) exponential synchronization. It extends the work in [2] where only two interconnected systems are discussed. We show that a very important tool to achieve synchronizing is to construct a symmetric covariant tensor field of order two which Lie derivative has to satisfy some specific properties.

The paper is organized as follows. Section III defines the synchronization problem studied here. Section III is devoted to establish necessary and sufficient conditions to the synchronization problem. Finally Section IV gives the conclusions.

Notations. The vector of all ones with a dimension $N$ is denoted by $\mathbb{1}_{N}$. We denote the identity matrix of dimension $n$ by $\operatorname{Id}_{n}$.

\section{PROBLEM DEFINITION}

\section{A. System description and communication topology}

In this note, we consider the problem of synchronizing $N$ identical nonlinear systems with $N \geq 2$. For every $i=$ $1, \ldots, N$, the $i$-th system $\Sigma_{i}$ is described by

$$
\dot{x}_{i}=f\left(x_{i}\right)+g\left(x_{i}\right) u_{i}, i=1, \ldots, N
$$

where $x_{i} \in \mathbb{R}^{n}, u_{i} \in \mathbb{R}^{p}$ and the functions $f$ and $g$ are assumed to be $C^{2}$. In this setting, all systems has the same drift vector field $f$ and the same control vector field $g$ : $\mathbb{R}^{n} \rightarrow \mathbb{R}^{n \times p}$, but not the same controls in $\mathbb{R}^{p}$. For simplicity of notation, we denote the complete state variables by $x=$ $\left[x_{1}^{\top}, \ldots, x_{N}^{\top}\right]^{\top}$ which is in $\mathbb{R}^{N n}$.

The synchronization manifold $\mathcal{D}$, where the state variables of different systems agree with each other, is defined by

$$
\mathcal{D}=\left\{\left(x_{1}, \ldots, x_{N}\right) \in \mathbb{R}^{N n} \mid x_{1}=x_{2} \cdots=x_{N}\right\} .
$$

For every $x$ in $\mathbb{R}^{N n}$, we denote the Euclidean distance to the set $\mathcal{D}$ by $|x|_{\mathcal{D}}$.

The communication graph $\mathcal{G}$, which is used for synchronizing the state through distributed control $u_{i}, i=1, \ldots N$, 
is assumed to be an undirected graph and is defined by $\mathcal{G}=(\mathcal{V}, \mathcal{E})$, where $\mathcal{V}$ is the set of $N$ nodes (where the $i$-th node is associated to the system $\Sigma_{i}$ ) and $\mathcal{E} \subset \mathcal{V} \times \mathcal{V}$ is a set of $M$ edges that define the pairs of communicating systems. Moreover we assume that the graph $\mathcal{G}$ is connected.

Let us, for every edge $k$ in $\mathcal{G}$ connecting node $i$ to node $j$, label one end (e.g., the node $i$ ) by a positive sign and the other end (e.g., the node $j$ ) by a negative sign. The incidence matrix $D$ that corresponds to $\mathcal{G}$ is an $N \times M$ matrix such that

$$
d_{i, k}= \begin{cases}+1 & \text { if node } i \text { is the positive end of edge } k \\ -1 & \text { if node } i \text { is the negative end of edge } k \\ 0 & \text { otherwise }\end{cases}
$$

Using $D$, the Laplacian matrix $L$ can be given by $L=D D^{\top}$ whose kernel, by the connectedness of $\mathcal{G}$, is spanned by $\mathbb{1}_{N}$. We will need the following lemma on the property of $L$ in our main result.

Lemma 1: Let $L=\left[\begin{array}{cc}L_{11} & L_{1,2: n} \\ L_{1,2: n}^{\top} & L_{2: n, 2: n}\end{array}\right]$ be a non-zero balanced Laplacian matrix associated to an undirected graph $\mathcal{G}$ where $L_{11}$ is a scalar. Then, the eigenvalues of the $(N-1) \times(N-1)$ matrix $\bar{L}:=L_{2: n, 2: n}-\mathbb{1}_{N-1} L_{1,2: n}$ are the same as the non-zero eigenvalues of $L$ with the same multiplicity. Moreover, if the graph is connected then $-\bar{L}$ is Hurwitz.

The proof of Lemma 1 can be found in Appendix A.

\section{B. Synchronization problem formulation}

Using the description of the interconnected systems via $\mathcal{G}$, the state synchronization control problem is defined as follows.

Definition 1: The control laws $u_{i}=\phi_{i}(x), i=1 \ldots, N$ solve the local uniform exponential synchronization problem of (1) if the following conditions hold:

1) For all non-communicating pair $(i, j)$ (i.e., $(i, j) \notin \mathcal{E}$ ), $\frac{\partial \phi_{i}}{\partial x_{j}}(x)=\frac{\partial \phi_{j}}{\partial x_{i}}(x)=0$

2) For all $x \in \mathcal{D}, \phi(x)=0$ (i.e., $\phi$ is zero on $\mathcal{D}$ ); and

3) The manifold $\mathcal{D}$ of the closed-loop system

$$
\dot{x}_{i}=f\left(x_{i}\right)+g\left(x_{i}\right) \phi_{i}(x), \quad i=1, \ldots, N
$$

is uniformly exponentially stable, i.e., there exist positive constants $r, k$ and $\lambda>0$ such that for all $x$ in $\mathbb{R}^{N n}$ satisfying $|x|_{\mathcal{D}}<r$,

$$
|X(x, t)|_{\mathcal{D}} \leq k \exp (-\lambda t)|x|_{\mathcal{D}},
$$

where $X(x, t)$ denotes the solution initiated from $x$, holds for all $t$ in the time domain of existence of solution.

When $r=\infty$, it is called the global uniform exponential synchronization problem.

In this definition, the condition 1) implies that the solution $u_{i}$ is a distributed control law that requires only a local state measurement from its neighbors in the graph $\mathcal{G}$.

An important feature of our study is that we focus on exponential stabilization of the synchronizing manifold. This allows us to rely on the study developed in [2] in which an infinitesimal characterization of exponential stability of a transverse manifold is given. As it will be shown in the following section this allows us to formalize some necessary and sufficient conditions in terms of matrix functions for a synchronizing control law to exist.

\section{NECESSARY AND SUFFICIENT CONDITION}

\section{A. Necessary conditions}

In [2], a first attempt has been made to give necessary conditions for the existence of an exponentially synchronizing control law for only two agents. In [3], the same problem has been addressed for $N$ agents but without any communication constraints (all agents can communicate with all others). In both cases, it is shown that assuming some bounds on derivatives of the vector fields the following two properties are necessary conditions.

IS Infinitesimal stabilizability. The couple $(f, g)$ is such that the $n$-dimensional manifold $\{\tilde{z}=0\}$ of the transversally linear system

$$
\begin{aligned}
\dot{\tilde{z}} & =\frac{\partial f}{\partial z}(z) \tilde{z}+g(z) \tilde{u} \\
\dot{z} & =f(z)
\end{aligned}
$$

with $\tilde{z}$ in $\mathbb{R}^{n}$ and $z$ in $\mathbb{R}^{n}$ is stabilizable by a state feedback that is linear in $\tilde{z}$ (i.e., $\tilde{u}=h(z) \tilde{z}$ for some function $h: \mathbb{R}^{n} \rightarrow \mathbb{R}^{p \times n}$ ).

CMF Control Matrix Function. For all positive definite matrix $Q \in \mathbb{R}^{n \times n}$, there exist a continuous function $P: \mathbb{R}^{n} \rightarrow \mathbb{R}^{n \times n}$ and strictly positive real numbers $p$ and $\bar{p}$ such that

$$
\underline{p} \operatorname{Id}_{n} \leq P(z) \leq \bar{p} \operatorname{Id}_{n}
$$

holds for all $z \in \mathbb{R}^{n}, P$ has a derivative $\mathfrak{d}_{f} P$ along $f$ in the following sense

$$
\mathfrak{d}_{f} P(z)=\lim _{h \rightarrow 0} \frac{P(Z(z, h))-P(z)}{h}
$$

where $Z(z, \cdot)$ is the solution to $4 \mathrm{~b}$ with an initial state $z$ in $\mathbb{R}^{n}$ so that the inequality

$$
v^{\top} \mathfrak{d}_{f} P(z) v+2 v^{\top} P(z) \frac{\partial f}{\partial z}(z) v \leq-v^{\top} Q v
$$

holds for all $(v, z)$ in $\mathbb{R}^{n} \times \mathbb{R}^{n}$ satisfying $v^{\top} P(z) g(z)=0$.

An important feature of properties IS and CMF comes from the fact that they are properties of each individual agent, independent of the network topology. The first one is a local stabilizability property. The second one establishes that there exists a symmetric covariant tensor field of order two denoted by $P$ whose Lie derivative satisfies a certain inequality in some specific directions. This type of condition can be related to the notion of control Lyapunov function which is a characterization of stabilizability as studied by Artstein in [5] or Sontag in [16]. This property can be regarding as an Artstein like condition. The dual of the CMF 
property has been thoroughly studied in [12] when dealing with an observer design ([12, Eq. (8)], see also [2] or [1]).

In this note, we show that properties IS and CMF are still necessary conditions if one considers a network of agents with a communication graph $\mathcal{G}$ as given in II-A. Hence, as this is already the case for linear system, we recover the paradigm which establishes that a necessary condition for synchronization is a stabilizability property for each individual agent. However, to obtain this property we need to restrict ourselves to a particular class of synchronizing control laws as given in the following theorem.

Theorem 1: Consider the interconnected systems in (1) with the communication graph $\mathcal{G}$ and assume that there exists a control law $u=\phi(x)$ where $\phi(x)=$ $\left[\begin{array}{lll}\phi_{1}^{\top}(x) & \ldots & \phi_{N}^{\top}(x)\end{array}\right]^{\top}$ that solves the local uniform exponential synchronization of (1). Assume that there exists a $C^{2}$ function $r: \mathbb{R}^{n} \rightarrow \mathbb{R}^{n}$ such that for all $x=\mathbb{1}_{N} \otimes z=$ $(z, \ldots z) \in \mathcal{D}$, with $z \in \mathbb{R}^{n}$ we have

$$
\frac{\partial \phi}{\partial x}(x)=r(z)^{\top} \otimes L
$$

where $L$ in $\mathbb{R}^{N \times N}$ is the Laplacian matrix associated to the graph. Assume moreover that $g$ is bounded and $f, g$ and the $\phi_{i}$ 's have bounded first and second derivatives and the closed-loop system is complete. Then properties IS and CMF hold.

Proof : The proof of IS is decomposed into the following two steps. In the first step, we show the stabilizability property for an $N n$-dimensional system which is established using the tools as developed in [2]. In the second step, employing the structure of the control law as given in Definition 11, we obtain the desired stabilizability property for the transversally linear system $4 \mathrm{a}$.

Step 1 : This part of the proof follows exactly the same steps as the ones presented in [3]. Let $e=$ $\left[\begin{array}{llll}e_{2}^{\top} & e_{3}^{\top} & \ldots & e_{N}^{\top}\end{array}\right]^{\top}$ with $e_{i}=x_{i}-x_{1}, i=2, \ldots N$, the closed-loop system (1) with the control law $\phi$ is given by

$$
\dot{e}=F\left(e, x_{1}\right), \dot{x}_{1}=G\left(e, x_{1}\right)
$$

with $e$ in $\mathbb{R}^{(N-1) n}, x_{1}$ in $\mathbb{R}^{n}$ and where

$$
\begin{aligned}
& F=\left[\begin{array}{llll}
F_{2}^{\top} & F_{3}^{\top} & \ldots & F_{N}^{\top}
\end{array}\right]^{\top} \\
& F_{i}\left(e, x_{1}\right)=f\left(x_{1}+e_{i}\right)-f\left(x_{1}\right) \\
& +g\left(x_{1}+e_{i}\right) \bar{\phi}_{i}\left(e, x_{1}\right)-g\left(x_{1}\right) \bar{\phi}_{1}\left(x_{1}\right), \\
& G\left(e, x_{1}\right)=f\left(x_{1}\right)+g\left(x_{1}\right) \bar{\phi}_{1}\left(e, x_{1}\right),
\end{aligned}
$$

where we have used the notation

$$
\bar{\phi}_{i}\left(e, x_{1}\right)=\phi_{i}\left(x_{1}, x_{1}+e_{2}, \ldots, x_{1}+e_{N}\right) .
$$

Note that we have

$$
\begin{aligned}
|e|^{2} & =\sum_{i=2}^{N}\left|x_{i}-x_{1}\right|^{2}, \\
& \leq(N-1)|x|_{\mathcal{D}}^{2},
\end{aligned}
$$

and

$$
\begin{aligned}
|x|_{\mathcal{D}}^{2} & \leq|e|^{2}+(N-1)\left|\sum_{i=1}^{N} \frac{x_{1}-x_{i}}{N}\right|^{2} \\
& \leq\left(1+\frac{N-1}{N^{2}}\right)|e|^{2} .
\end{aligned}
$$

Hence, if we denote $E\left(e, x_{1}, \cdot\right)$ the $e$ components of the solution to 9 , then 3 implies for all $\left(e, x_{1}\right)$ in $\mathbb{R}^{(N-1) n} \times$ $\mathbb{R}^{n}$

$$
\begin{aligned}
& \left|E\left(e, x_{1}, t\right)\right| \\
& \leq \sqrt{(N-1)\left(1+\frac{N-1}{N^{2}}\right)} k \exp (-\lambda t)|e| .
\end{aligned}
$$

It follows that the manifold $e=0$ is locally uniformly (in $x_{1}$ ) exponentially stable for $(9$. We conclude with [2, Prop. 1] that the so-called Property UES-TL is satisfied. Moreover, we conclude that there exist strictly positive real numbers $\widetilde{k}$ and $\tilde{\lambda}$ such that any solution $(\widetilde{E}(\widetilde{e}, z, \cdot), Z(z, \cdot))$ to

$$
\dot{\widetilde{e}}=\frac{\partial F}{\partial \widetilde{e}}(0, z) \widetilde{e}, \dot{z}=G(0, z)
$$

with $\widetilde{e}$ in $\mathbb{R}^{(N-1) n}$ and $z$ in $\mathbb{R}^{n}$ satisfies

$$
|\widetilde{E}(\widetilde{e}, z, t)| \leq \widetilde{k} \exp (-\tilde{\lambda} t)|\widetilde{e}|
$$

for all $(\widetilde{e}, z, t)$ in $\mathbb{R}^{(N-1) n} \times \mathbb{R}^{n} \times \mathbb{R}_{\geq 0}$.

By the definition of $\bar{\phi}$ in $\sqrt{13}$, it follows that in the manifold where $e=0$, we have that $\bar{\phi}_{i}\left(0, x_{1}\right)=0$. This implies that for every $i=1, \ldots, N$,

$$
\begin{aligned}
& \frac{\partial F_{i}}{\partial \widetilde{e}_{i}}(0, z)=\frac{\partial f}{\partial z}(z) \\
& \quad+g(z)\left[\frac{\partial \bar{\phi}_{i}}{\partial \widetilde{e}_{i}}(0, z)-\frac{\partial \bar{\phi}_{1}}{\partial \widetilde{e}_{i}}(0, z)\right]
\end{aligned}
$$

and for all $j \neq i$,

$$
\frac{\partial F_{i}}{\partial \widetilde{e}_{j}}(0, z)=g(z)\left[\frac{\partial \bar{\phi}_{i}}{\partial \widetilde{e}_{j}}(0, z)-\frac{\partial \bar{\phi}_{1}}{\partial \widetilde{e}_{j}}(0, z)\right] .
$$

Hence, this yields

$$
\begin{aligned}
\dot{\tilde{e}}_{i}=\frac{\partial F_{i}}{\partial \widetilde{e}}(0, z) \widetilde{e}= & \frac{\partial f}{\partial z}(z) \widetilde{e}_{i} \\
& +g(z)\left[\frac{\partial \bar{\phi}_{i}}{\partial \widetilde{e}}(0, z)-\frac{\partial \bar{\phi}_{1}}{\partial \widetilde{e}}(0, z)\right] \widetilde{e}
\end{aligned}
$$

Step 2: We will now show that $4 \mathrm{a}$ is stabilizable by a state feedback that is linear in $\tilde{z}$. In particular, the stabilizing control law for 4 a will be given by

$$
\tilde{u}=\nu r(z)^{\top} \tilde{z}
$$

where $\nu$ is a real non-zero eigenvalue of the matrix $L_{2: n, 2: n}-$ $\mathbb{1}_{N-1} L_{1,2: n}=: \bar{L}$, where $L=\left[\begin{array}{cc}L_{11} & L_{1,2: n} \\ L_{1,2: n}^{\top} & L_{2: n, 2: n}\end{array}\right]$ is the Laplacian of $\mathcal{G}$. By Lemma 1, all eigenvalues of $\bar{L}$ are the same as the non-zero eigenvalues of $L$ (which are all positive) with the same multiplicity and $-\bar{L}$ is Hurwitz. 
Let $v=\left(v_{2}, \ldots, v_{N}\right)$ in $\mathbb{R}^{N-1}$ be a left eigenvector of $\bar{L}$ associated to $\nu$, i.e.

$$
v^{\top} \bar{L}=\nu v^{\top}
$$

By using $\Pi=\operatorname{Id}_{n} \otimes v^{\top}$ as a mapping from $\mathbb{R}^{(N-1) n}$ to $\mathbb{R}^{n}$, we will show that the image of the solution to 16 are solution to (4a) with the control law 20). Indeed, by denoting $\tilde{z}=\Pi \tilde{e}$ and by using $[16$, we have

$$
\begin{aligned}
\dot{\tilde{z}}= & \overbrace{i=2}^{N} v_{i} \widetilde{e}_{i}=\frac{\partial f}{\partial z}(z) \sum_{i=2}^{N} v_{i} \widetilde{e}_{i} \\
& \quad+g(z) \sum_{i=2}^{N} v_{i}\left[\frac{\partial \bar{\phi}_{i}}{\partial \widetilde{e}}(0, z)-\frac{\partial \bar{\phi}_{1}}{\partial \widetilde{e}}(0, z)\right] \widetilde{e} \\
= & \frac{\partial f}{\partial z}(z) \sum_{i=2}^{N} v_{i} \widetilde{e}_{i} \\
& \quad+g(z) \sum_{i=2}^{N} v_{i} \sum_{j=2}^{N}\left[L_{i j}-L_{1 j}\right] r(z)^{\top} \widetilde{e}_{j} \\
= & \frac{\partial f}{\partial z}(z) \sum_{i=2}^{N} v_{i} \widetilde{e}_{i} \\
& \quad+g(z) r(z)^{\top} v^{\top}\left(L_{2: n, 2: n}-\mathbb{1}_{N-1} L_{1,2: n}\right) \widetilde{e} \\
= & {\left[\frac{\partial f}{\partial z}(z)+g(z) \nu r(z)^{\top}\right] \tilde{z} . }
\end{aligned}
$$

Denote the solution of the above equation by $\tilde{Z}(\tilde{z}, z, t)$. Since $\tilde{Z}(\tilde{z}, z, t)=\Pi \widetilde{E}(\widetilde{e}, z, t)$, it follows from 17 that

$$
|\tilde{Z}(\tilde{z}, z, t)| \leq|v| \widetilde{k} \exp (-\widetilde{\lambda} t)|\widetilde{e}|
$$

This proves that the property IS holds.

Proof of CMF : To prove CMF, we use the property IS and [2, Proposition 2]. Indeed, note that if we consider another system with state $(\tilde{z}, z)$ in $\mathbb{R}^{n} \times \mathbb{R}^{n}$ that is described by

$$
\dot{\tilde{z}}=\bar{F}(\tilde{z}, z), \dot{z}=f(z)
$$

with $\bar{F}(\tilde{z}, z)=f(\tilde{z}+z)-f(z)+g(z) \nu r(z)^{\top} \tilde{z}, \nu \in \mathbb{R}$, it follows from IS and [2, Proposition 2] that there exist a function $P: \mathbb{R}^{n} \rightarrow \mathbb{R}^{n}$ such that CMF holds. In particular, we have that, for all $(v, z)$ in $\mathbb{R}^{n} \times \mathbb{R}^{n}$,

$$
\begin{aligned}
v^{\top} \mathfrak{d}_{f} P(z) & \\
& +2 v^{\top} P(z)\left(\frac{\partial f}{\partial z}(z)+g(z) \nu r(z)^{\top}\right) v \leq-v^{\top} Q v
\end{aligned}
$$

which implies that 7) holds when $v^{\top} P(z) g(z)=0$.

\section{B. A sufficient condition}

The interest that we have in the Property CMF given in Subsection III-A is to use the symmetric covariant tensor $P$ in the design of a local synchronizing control law. Indeed, following one of the main results in [2], we get the following sufficient condition for the solvability of (local) uniform exponential synchronization problem. The first assumption is that, up to a scaling factor, the control vector field $g$ is a gradient field with $P$ as a Riemannian metric.

Theorem 2 (Local sufficient condition): Assume that $g$ is bounded and that $f$ and $g$ have bounded first and second derivatives. Assume that there exists a $C^{2}$ function $P: \mathbb{R}^{n} \rightarrow$ $\mathbb{R}^{n \times n}$ with a bounded derivative that satisfies the following two conditions.

1. There exist a $C^{2}$ function $U: \mathbb{R}^{n} \rightarrow \mathbb{R}$ which has bounded first and second derivatives, and a $C^{1}$ function $\alpha: \mathbb{R}^{n} \rightarrow \mathbb{R}^{p}$ which has bounded first and second derivative such that

$$
\frac{\partial U}{\partial z}(z)^{\top}=P(z) g(z) \alpha(z)
$$

holds for all $z$ in $\mathbb{R}^{n}$; and

2. There exist a positive definite matrix $Q$ and positive constants $\underline{p}, \bar{p}$ and $\rho>0$ such that $(5)$ and

$$
v^{\top} \mathfrak{d}_{f} P(z) v+2 v^{\top} P(z) \frac{\partial f}{\partial z}(z) v-\rho\left|\frac{\partial U}{\partial z}(z) v\right|^{2} \leq-v^{\top} Q v,
$$

holds for all $(z, v)$ in $\mathbb{R}^{n} \times \mathbb{R}^{n}$.

Then, given a connected graph $\mathcal{G}$ with associated Laplacian matrix $L=\left(L_{i j}\right)$, there exists a constant $\underline{\ell}$ such that the control laws $u=\phi(x)$ with $\phi=\left[\begin{array}{lll}\phi_{1}^{\top} & \ldots & \phi_{N}^{\top}\end{array}\right]^{\top}$ given by

$$
\phi_{i}(x)=-\ell \alpha\left(x_{i}\right) \sum_{j=1}^{N} L_{i j} U\left(x_{j}\right)
$$

and $\ell \geq \underline{\ell}$ solves the local uniform exponential synchronization of (1).

Proof : First of all, note that the control law $\phi$ satisfies the condition 1) and 2) in Definition 1. Indeed, for all $x=$ $\mathbb{1}_{N} \otimes z=(z, \ldots, z)$ in $\mathcal{D}$ and for all $(i, j)$

$$
\frac{\partial \phi_{i}}{\partial x_{j}}(x)=-\ell \alpha(z) L_{i j} \frac{\partial U}{\partial z}(z)
$$

Hence, for all $x=\mathbb{1}_{N} \otimes z$ in $\mathcal{D}$, we get

$$
\frac{\partial \phi}{\partial x}(x)=-\ell \alpha(z) \frac{\partial U}{\partial z}(z) \otimes L
$$

It remains to prove that the manifold $\mathcal{D}$ of the closed-loop system is locally exponentially stable.

As in the proof of Theorem 11, let us denote $e=$ $\left(e_{2}, \ldots, e_{N}\right)$ with $e_{i}=x_{1}-x_{i}$. Note that the closed-loop system may be rewritten as in 9 with the vector fields $F$ and $G$ as defined in $10-12$ with $\phi$ as the control law.

The rest of the proof is to apply [2, Proposition 3]. For this purpose, we need to show that for closed-loop system (10)(12) the property ULMTE introduced in [2] is satisfied. More precisely, we need to show that there exists a symmetric covariant 2-tensor field $P_{N}: \mathbb{R}^{n} \rightarrow \mathbb{R}^{(N-1) n \times(N-1) n}$ which satisfies [2, Ineq. (9)].

By the assumption on the graph being connected and together with Lemma 1, we have that the matrix $A=-\left(L_{2: n, 2: n}-\mathbb{1}_{N-1} L_{1,2: n}\right)$ is Hurwitz. Let $S$ in 
$\mathbb{R}^{(N-1) \times(N-1)}$ be a positive definite matrix solution to the Lyapunov equation

$$
S A+A^{\top} S \leq-\nu S
$$

where $\nu$ is a positive real number.

Consider the function $P_{N}: \mathbb{R}^{n} \rightarrow \mathbb{R}^{(N-1) n \times(N-1) n}$ defined as

$$
P_{N}(z)=S \otimes P(z)
$$

Note that we have $G(0, z)=f(z)$. Moreover we have

$$
\mathfrak{d}_{G(0, z)} P_{N}(z)=S \otimes \mathfrak{d}_{f} P(z) .
$$
that

Note that with properties (18), (19) and (26), it follows

$$
\begin{aligned}
\frac{\partial F}{\partial \widetilde{e}}(0, z)=\operatorname{Id}_{N-1} \otimes & \frac{\partial f}{\partial z}(z) \\
& +\ell A \otimes\left(\alpha(z) g(z) \frac{\partial U}{\partial z}(z)\right)
\end{aligned}
$$

Hence,

$$
\begin{aligned}
\mathfrak{d}_{G(0, z)} P_{N}(z)+P_{N}(z) \frac{\partial F}{\partial \widetilde{e}}(0, z)+\frac{\partial F}{\partial \widetilde{e}}(0, z)^{\top} P_{N}(z) \\
=S \otimes\left(\mathfrak{d}_{f} P(z)+P(z) \frac{\partial f}{\partial z}(z)+\frac{\partial f}{\partial z}(z)^{\top} P(z)\right) \\
+\ell\left(S A+A^{\top} S\right) \otimes\left(\frac{\partial U}{\partial z}(z)^{\top} \frac{\partial U}{\partial z}(z)\right)
\end{aligned}
$$

With 27) and 23, this implies that

$$
\begin{aligned}
& \mathfrak{d}_{f} P_{N}(z)+ P_{N}(z) \frac{\partial F}{\partial \widetilde{e}}(0, z)+\frac{\partial F}{\partial \widetilde{e}}(0, z)^{\top} P_{N}(z) \\
& \leq S \otimes\left(-Q+(\rho-\ell \nu) \frac{\partial U}{\partial z}(z)^{\top} \frac{\partial U}{\partial z}(z)\right)
\end{aligned}
$$

Hence, by choosing $\underline{\ell}>\frac{\rho}{\nu}$, [2, Inequality (9)] holds. The last part of the proof is to make sure that the vector field $F$ has bounded first and second derivatives and that the vector field $G$ has bounded first derivative. Note that by employing the bounds on the functions $P, f, g, \alpha$ and their derivatives, the result follows immediately from [2, Proposition 3]. Hence $e=0$ is (locally) exponentially stable manifold for system (10)-12] in closed loop with the control (24). With inequalities (14) and (15), it implies that inequality (3) holds for $r$ sufficiently small.

Remark 1: Note that in [3] with an extra assumption related to the metric (the level sets of $U$ are totally geodesic set with respect to the Riemanian metric obtained from $P$ ), it is shown that global synchronization may be achieved when all agents are connected to all other. It is still an open question to know if global synchronization may be achieved in the communication topology constraint context.

\section{Illustrative example}

As an illustrative example, consider the case in which the vector fields $f$ and $g$ are given by

$$
\dot{z}=f(z)+g(z) u
$$

with

$$
\begin{gathered}
f(z)=\left[\begin{array}{c}
-z_{1}+\sin \left(z_{2}\right) \cos \left(z_{1}\right)+z_{2} \\
0
\end{array}\right], \\
g(z)=\left[\begin{array}{c}
0 \\
2+\sin \left(z_{1}\right)
\end{array}\right]
\end{gathered}
$$

We will now show that the above system satisfy the hypotheses in Theorem 2. Consider a constant matrix $P$ given by

$$
P=\left(\begin{array}{ll}
2 & 1 \\
1 & 2
\end{array}\right)
$$

It can be checked that by taking

$$
U(z)=z_{1}+2 z_{2}
$$

Eq. 22 holds with $\alpha=\frac{1}{2+\sin \left(z_{1}\right)}$. Using the above $U$, it follows that

$$
v^{\top} \frac{\partial U}{\partial z}(z)=0 \Leftrightarrow v_{1}+2 v_{2}=0
$$

in which case, by taking $v=\left[\begin{array}{c}-2 \\ 1\end{array}\right] s$ where $s \in \mathbb{R}$, Eq. 23 becomes

$$
\begin{aligned}
& {\left[\begin{array}{ll}
-2 & 1
\end{array}\right] P \frac{\partial f}{\partial z}(z)\left[\begin{array}{c}
-2 \\
1
\end{array}\right]=-3\left[-2 \frac{\partial f_{1}}{\partial z_{1}}+\frac{\partial f_{1}}{\partial z_{2}}\right]} \\
& =-3\left[-2\left(-1+\sin \left(z_{2}\right) \sin \left(z_{1}\right)\right)-\cos \left(z_{1}\right) \cos \left(z_{2}\right)+1\right] \\
& \leq-3 \text {. }
\end{aligned}
$$

Hence, 23 holds by taking $\rho$ sufficiently large.

The function $\frac{\partial f}{\partial z}(z)$ being periodic in $z_{a 1}$ and $z_{a 2}$ we can assume that $z_{a 1}$ and $z_{a 2}$ are in compact subset. This implies employing some variation on Finsler lemma that there exists $\rho$ and $Q$ such that inequality (23) holds. Hence from Theorem 2, the control law given in 24) solves the local exponential synchronization problem for the $N$ identical systems that exchange information via any undirected communication graph $\mathcal{G}$ which is connected.

\section{CONCLUSION}

In this paper, based on recent results in [2], we have presented necessary and sufficient condition for the solvability of local exponential synchronization of $N$ identical affine nonlinear systems through a distributed control law. In particular, we have shown that the necessary condition is linked to the infinitesimal stabilizability of the individual system and is independent of the network topology. The existence of a symmetric covariant tensor of order two, as a result of the infinitesimal stabilizability, has allowed us to design a distributed synchronizing control law.

\section{APPENDIX}

\section{A. Proof of Lemma 1}

From the property of Laplacian matrix, the eigenvalues of $L$ are real and satisfy $0=\lambda_{1} \leq \lambda_{2} \leq \ldots \leq \lambda_{N}$. Let us take the non-zero eigenvalue $\nu>0$ of $L$ and its corresponding eigenvector $v$ in $\mathbb{R}^{N}$. Note that we can decompose

$$
v=\left[\begin{array}{c}
v_{a} \\
v_{b}
\end{array}\right], L=\left[\begin{array}{cc}
L_{11} & L_{1,2: n} \\
L_{1,2: n}^{\top} & L_{2: n, 2: n}
\end{array}\right]
$$


with $v_{a}$ and $L_{11}$ in $\mathbb{R}$. It follows that

$$
\begin{aligned}
L_{11} v_{a}+L_{1,2: n} v_{b} & =\nu v_{a} \\
L_{1,2: n}^{\top} v_{a}+L_{2: n, 2: n} v_{b} & =\nu v_{b} .
\end{aligned}
$$

Moreover, since $\mathbb{1}_{N}$ is an eigenvector associated to the eigenvalue 0 ,

$$
\begin{array}{r}
L_{11}+L_{1,2: n} \mathbb{1}_{N-1}=0 \\
L_{1,2: n}^{\top}+L_{2: n, 2: n} \mathbb{1}_{N-1}=0
\end{array}
$$

Consider now a vector in $\mathbb{R}^{N-1}$ defined by

$$
\tilde{v}=v_{b}-\mathbb{1}_{N-1} v_{a}
$$

Note that $\tilde{v}$ is non zero since $v$ is not colinear to $\mathbb{1}_{N}$. By a routine algebraic computation, it follows that this vector satisfies

$$
\begin{gathered}
{\left[L_{2: n, 2: n}-\mathbb{1}_{N-1} L_{1,2: n}\right] \tilde{v}=L_{2: n, 2: n} v_{b}-\mathbb{1}_{N-1} L_{1,2: n} v_{b}} \\
+\left[\mathbb{1}_{N-1} L_{1,2: n} \mathbb{1}_{N-1}-L_{2: n, 2: n} \mathbb{1}_{N-1}\right] v_{a} \\
=\nu v_{b}-L_{1,2: n}^{\top} v_{a} \\
-\mathbb{1}_{N-1} L_{1,2: n} v_{b}+\left[-\mathbb{1}_{N-1} L_{11}+L_{1,2: n}\right] v_{a} \\
=\nu \tilde{v} .
\end{gathered}
$$

This shows that $\tilde{v}$ is an eigenvector with the same non-zero eigenvalue of $L$. It proves the first claim of the lemma.

Note that the multiplicity of the eigenvalue $\nu$ is the same for both matrices. Also, if the graph is connected, then the 0 eigenvalue of the Laplacian matrix $L$ is of multiplicity 1 and the other eigenvalues are positive and distinct. Hence the matrix $-\bar{L}$ is Hurwitz.

\section{REFERENCES}

[1] V. Andrieu, G. Besancon, and U. Serres. Observability necessary conditions for the existence of observers. In Proc. of the 52nd IEEE Conference on Decision and Control, 2013.

[2] V. Andrieu, B. Jayawardhana, and L. Praly. On transverse exponential stability and its use in incremental stability, observer and synchronization. In Proc. of the 52nd IEEE Conference on Decision and Control, 2013.

[3] V. Andrieu, B. Jayawardhana, and L. Praly. Transverse exponential stability and applications. Under review, 2015.

[4] D. Angeli. Further results on incremental input-to-state stability. Automatic Control, IEEE Transactions on, 54(6):1386-1391, 2009.

[5] Z. Arststein. Stabilization with relaxed controls. Nonlinear analysis, 7(11):1163-1173, 1983

[6] C. De Persis and B. Jayawardhana. Coordination of passive systems under quantized measurements. SIAM Journal Control and Optimization, 50(6):3155-3177, 2012.

[7] C. De Persis and B. Jayawardhana. On the internal model principle in formation control and in output synchronization of nonlinear systems. In Proc. 51st IEEE Conference on Decision and Control, 2012.

[8] C. De Persis and B. Jayawardhana. On the Internal Model Principle in the Coordination of Nonlinear Systems. IEEE Transactions on Control of Network Systems, 1(3):272-282, 2014.

[9] B.C. Goodwin. Oscillatory behaviour in enzymatic control processes. Advances in Enzyme Regulation, 3:425-428, 1965.

[10] A. Hamadeh, G-B. Stan, and J. Goncalves. Contructive synchronization of networked feedback systems. In Proc. 49th IEEE Conf. Decision and Control, pages 6710-6715, 2010.

[11] A. Hamadeh, G-B. Stan, R. Sepulchre, and J. Goncalves. Global state synchronization in networks of cyclic feedback systems. IEEE Trans. Automatic Control, 57(2):478-483, 2012.

[12] R.G. Sanfelice and L. Praly. Convergence of nonlinear observers on $\mathbb{R}^{n}$ with a riemannian metric (part i). IEEE Trans. Automatic Control, 57(7):1709-1722, 2012.
[13] A. Sarlette, R. Sepulchre, and N.E. Leonard. Autonomous rigid body attitude synchronization. Automatica, 45(2):572-577, 2009.

[14] L. Scardovi, M. Arcak, and E.D. Sontag. Synchronization of interconnected systems with applications to biochemical networks: An inputoutput approach. IEEE Trans. Automatic Control, 55(6):1367-1379, 2010.

[15] L. Scardovi and R. Sepulchre. Synchronization in networks of identical linear systems. Automatica, 45(11):2557-2562, 2009.

[16] E.D. Sontag. A Lyapunov-Like characterization of asymptotic controllability. SIAM Journal on Control and Optimization, 21:462-471, 1983.

[17] P. Wieland, R. Sepulchre, and F. Allgöwer. An internal model principle is necessary and sufficient for linear output synchronization. Automatica, 47(5):1068-1074, 2011. 